\title{
UCRL-JRNL-200939
}

\section{Static High-Pressure Structural Studies on Dy to 119 GPa}

J. R. Patterson, C. K. Saw, and J. Akella

November 14, 2003

Journal of Applied Physics 
This document was prepared as an account of work sponsored by an agency of the United States Government. Neither the United States Government nor the University of California nor any of their employees, makes any warranty, express or implied, or assumes any legal liability or responsibility for the accuracy, completeness, or usefulness of any information, apparatus, product, or process disclosed, or represents that its use would not infringe privately owned rights. Reference herein to any specific commercial product, process, or service by trade name, trademark, manufacturer, or otherwise, does not necessarily constitute or imply its endorsement, recommendation, or favoring by the United States Government or the University of California. The views and opinions of authors expressed herein do not necessarily state or reflect those of the United States Government or the University of California, and shall not be used for advertising or product endorsement purposes. 


\title{
Static High-Pressure Structural Studies on Dy to 119 GPa
}

\author{
Reed Patterson, Cheng K. Saw and Jagannadham Akella \\ Lawrence Livermore National Laboratory, Livermore CA, 94551
}

\begin{abstract}
Structural phase transitions in the rare-earth metal Dysprosium have been studied in a Diamond Anvil Cell (DAC) to $119 \mathrm{GPa}$ by x-ray diffraction. Four transformations following the sequence hcp $\rightarrow$ Sm-type $\rightarrow$ dhcp $\rightarrow$ hR24 (hexagonal) $\rightarrow$ bcm (monoclinic) are observed at 6, 15, 43, and $73 \mathrm{GPa}$ respectively. The hexagonal to monoclinic transformation is accompanied by a $6 \%$ reduction in volume, which is attributed to delocalization of the $4 \mathrm{f}$ electrons, similar to that seen in $\mathrm{Ce}, \mathrm{Pr}$, and $\mathrm{Gd}$.
\end{abstract}

\section{INTRODUCTION}

The compression of rare earth metals is known to induce both structural and electronic transformations. The trivalent lanthanides (La through Lu excepting Eu and $\mathrm{Yb}$ ) have been shown to follow a crystal structure sequence with decreasing atomic number or increasing pressure: hcp $\rightarrow$ Sm-type $\rightarrow$ dhcp $\rightarrow$ fcc. ${ }^{1}$ This structural sequence is attributed to the promotion of s electrons to the d-band with increasing pressure. In these pressure regimes, the felectrons do not contribute to bonding. Further compression studies of the rare earth metals have added a distorted fcc (dfcc) phase to this pressure sequence. ${ }^{2}$ Still further compression of several rare earth elements has resulted in a transition to a lower symmetry phase, which may be accompanied by a significant volume change. One explanation is that this transition is a Mott 
transition, ${ }^{3}$ in which the f electrons become delocalized and now participate in the metallic bond. Although the delocalization of the f electrons is the generally accepted explanation for the collapsed phases, the phase itself depends on the element studied, with at least monoclinic and orthorhombic phases having been observed.

Dy crystallizes in an hcp structure at ambient conditions with $\mathrm{a}=3.5918$ and $\mathrm{c}=5.6518$

$\AA^{4}$ Dy is a trivalent rare earth metal with ambient pressure electronic configuration of $(\mathrm{spd})^{3} 4 \mathrm{f}^{9}$ with 3 eectrons in the spd conduction band and 9 electrons in the localized $4 \mathrm{f}$ shell. The application of high pressure is expected to cause s-d electron transfer in the spd conduction band and at sufficiently high-pressure cause an eventual delocalization of the $4 \mathrm{f}$ shell. Previous studies on Dy have shown it to follow the typical rare earth structural sequence through the dfcc phase. Großhans ${ }^{5}$ found pressure induced transformations occurring at about 8, 17, and 43 GPa for the hcp $\rightarrow$ Sm-type, Sm-type $\rightarrow$ dhcp, and dhcp $\rightarrow$ dfcc transitions, respectively. Akella et al. ${ }^{6}$ also observed similar transition pressures, and find the dfcc phase to be stable up to $57 \mathrm{GPa}$. Neither observes a pure fcc phase. In the rare earth metals $\mathrm{Pr}, \mathrm{Nd}, \mathrm{Sm}$, and $\mathrm{Gd}$, the dfcc phase precedes a lower symmetry phase that is associated with $\mathrm{f}$ electron delocalization. In view of these observations Dy is a good candidate for an f-shell delocalization transition at pressures greater than $57 \mathrm{GPa}$. In the present work, we examine the post fcc phases of Dy and the pressure-volume EOS to $119 \mathrm{GPa}$. Our high pressure studies are motivated by structural transformations in Dy above $57 \mathrm{GPa}$ and the correlation of these transformations to the $4 \mathrm{f}$-shell delocalization phenomenon, as well as comparisons with phase changes in neighboring rare earth metals at high compressions. 


\section{EXPERIMENT}

We conducted in-situ energy dispersive x-ray diffraction measurements on Dy to 119 GPa. X-ray diffraction spectra were collected on beamline X-17C at the National Synchrotron Light Source of Brookhaven National Laboratory. Typical collimated beam size is on the order of $10 \mu \mathrm{m}$ by $12 \mu \mathrm{m}$ from a $2.5 \mathrm{GeV}$ beam. Dy of $99.99 \%$ purity was purchased from the Materials Preparation Center at Ames Laboratory. Prior to loading, the sample was scraped and then cleaned with alcohol in order to expose clean metal and reduce the amount of possible oxide. A Mao-Bell type DAC was prepared with type Ia diamonds having a $7.5^{\circ}$ bevel from a $300 \mu \mathrm{m}$ culet to a 50-70 $\mu \mathrm{m}$ central flat. The sample was loaded into a 30-60 $\mu \mathrm{m}$ chamber drilled in a pre-indented tool-steel gasket. $\mathrm{Cu}$ was loaded in the sample chamber as a pressure standard, and no pressure medium was employed in order to maximize sample volume and avoid any chemical reactions between the sample and pressure medium. Pressure was calibrated against the internal $\mathrm{Cu}$ standard using the Vinet $\operatorname{EOS}^{7,8}$,

$$
\ln H=\ln K_{0}+\eta(1-x)+\beta(1-x)^{2}
$$

where $H=P x^{2} / 3(1-x), \eta=3 / 2\left(K_{0}^{\prime}-1\right)$, and $x=\left(V / V_{0}\right)^{1 / 3}$. Experimental pressures were calculated using the values $K_{0}=143.66, \eta=4.3566$, and $\beta=13.774$, from the shock data for copper $^{9}$.

\section{RESULTS AND DISCUSSION}


EDX spectra of the starting material at ambient conditions show a clean hcp structure with no detectable oxide peaks (Fig. 1). Measured lattice parameters of $\mathrm{a}=3.5885$ and $\mathrm{c}=$ $5.6434 \AA$ are consistent with the literature values. ${ }^{4}$ On compression, we observe the standard rare earth crystal sequence in good agreement with the literature. Fig. 1 shows the EDX spectra for the different phases obtained on compression. Following the initial hcp spectra, we observe the Sm-type structure at $6.0 \mathrm{GPa}$ and then dhcp at $15.1 \mathrm{GPa}$. Although we never observe a pure fcc phase, the fcc stability region may lie between our pressure steps (37 to $43 \mathrm{GPa}$ ). The fcc stability field is known to narrow considerably in heavy rare earth metals and our high pressure results suggest that fcc stability field in Dy is less than 6 GPa. . Upon increasing pressure to 43.0 $\mathrm{GPa}$, we find the completion of the dhcp $\rightarrow$ dfcc transition.

The structure of the dfcc phase has been the subject of some debate. A careful refinement of the dfcc phase in Pr found a hexagonal cell with $\mathrm{Z}=24$ (denoted hR24) to be the best fit. ${ }^{10} \mathrm{hR} 24$ belongs to group R-3m with atoms occupying the $6 \mathrm{c}$ and $18 \mathrm{~h}$ sites. Table I gives the indexing for hR24 at $61.6 \mathrm{GPa}$, with lattice parameters $\mathrm{a}=5.7032$ and $\mathrm{c}=13.9721$. The atomic positions given by Hamaya et al. ${ }^{10}$ were used for structure assignment, and not refined further. The measured and calculated d-spacings are in good agreement, however, evidence of preferred orientation is seen in the differences in the measured and calculated intensities. Pure hR24 exists until at least $69.4 \mathrm{GPa}$, followed by a phase transformation at 73.3 $\pm 3.9 \mathrm{GPa}$. We find a simple body centered monoclinic $(\mathrm{bcm})$ structure best explains the spectra acquired above $74 \mathrm{GPa}$.

$\mathrm{Bcm}$ phases are also observed in $\mathrm{Ce}\left(\alpha^{\prime \prime}-\mathrm{Ce}\right)^{11}$ and $\mathrm{Gd}^{12}$ under pressure. Our data more closely resemble Gd as indicated by the indexing shown in Fig. 2. The slight variation in the bcm cells of Ce and Dy may result from the intermediate hR24 phase found in Dy but not in Ce. 
Gd also has an intermediate distorted fcc phase although it was indexed as 6-layered hexagonal. ${ }^{12}$ A mixture of hR24 and bcm is observed until 81.8 GPa. Fig. 2 shows the bcm phase at $81.8 \mathrm{GPa}$ as well as at $118.6 \mathrm{GPa}$, the highest pressure attained in this study. The bcm phase has lattice parameters $\mathrm{a}=2.6932, \mathrm{~b}=2.1728$, and $\mathrm{c}=4.3534 \AA$, with $\beta=92.61^{\circ}$ at 118.6 GPa. The d-spacings are given in Table II. Calculated bcm structures show a large (110) peak. We observe a weak (110) peak at $81.8 \mathrm{GPa}$, most likely due to sample texturing, and this peak is totally obscured at $118.6 \mathrm{GPa}$ by the diamond absorption edge.

The pressure-volume EOS for Dy is shown in Fig. 3. The solid curve is a $3^{\text {rd }}$ order BirchMurnaghan fit, given by

$$
P=\frac{3}{2} K_{0}\left(x^{7 / 3}-x^{5 / 3}\right)\left\{1+\frac{3}{4}\left(K_{0}^{\prime}-4\right)\left(x^{2 / 3}-1\right)\right\}, x=\left(\frac{V_{0}}{V}\right) .
$$

$\mathrm{V}_{0}$ is the volume at ambient conditions, $\mathrm{K}_{0}$ is the isothermal compressibility, and $\mathrm{K}_{0}$ ' is the first derivative of $\mathrm{K}_{0}$. The compression of Dy is continuous for all structures through hR24. The $\mathrm{hR} 24 \rightarrow$ bcm transition, however, is accompanied by a $6 \%$ volume change, following which Dy becomes much less compressible. Previous studies ${ }^{5}$ found $\mathrm{K}_{0}=37.8(9) \mathrm{GPa}$ and $\mathrm{K}_{0}{ }^{\prime}=2.8(1)$ GPa. We find $\mathrm{K}_{0}=36.22 \mathrm{GPa}$ and $\mathrm{K}_{0}{ }^{\prime}=3.29 \mathrm{GPa}$ for the pre collapse phases, and $\mathrm{K}_{0}=24.08$ $\mathrm{GPa}$ and $\mathrm{K}_{0}{ }^{\prime}=4.06 \mathrm{GPa}$ for the $\mathrm{bcm}$ phase.

Of the rare earth elements in which low symmetry phases have been observed to follow the dfcc phase, Ce, Pr, and Gd show discontinuous transitions, while Nd and Sm do not. These phase transitions have been associated with $\mathrm{f}$ electron participation in bonding, and it is known from the actinide elements that bonding $\mathrm{f}$ electrons favor low symmetry structures. For the lanthanides, as pressure increases, $\mathrm{f}$ electrons begin to participate in bonding, therefore at some 
point in the compression of the rare earth metals, it will be energetically favorable to transform to a low symmetry structure. The bcm phase of Dy (similar to that found in Gd) has $c>b$, while the bcm phase for Ce has b > c. Ce undergoes an isostructural phase transition ( $f c c \rightarrow f c c$ '), which is associated with $\mathrm{f}$ electron delocalization, before transforming to bcm. Dy and Gd transform from dfcc phases to bcm, with this transition being the f electron delocalization. The non-isostructural nature of this transition has been attributed to the interactions of multiple $f$ electrons ${ }^{12}$. Thus the differences in the bcm cells for Dy and Ce may be a result of the transformation path from the preceding phases, as well as contribution from bonding f electrons.

\section{CONCLUSIONS}

In summary, the dfcc phase of Dy has been indexed to an hR24 structure in the pressure range between $43 \mathrm{GPa}$ and $73 \mathrm{GPa}$. In addition, Dy is shown to have a volume change of $6 \%$ at 73.3 GPa, corresponding to an $\mathrm{hR} 24 \rightarrow \mathrm{bcm}$ transition. We attribute this transition to the delocalization of the $4 \mathrm{f}$ electrons. The body centered monoclinic phase (similar to that observed in $\mathrm{Gd}$ ) is seen to be stable to $118.6 \mathrm{GPa}$, at which the volume compression is $\mathrm{VlV}_{0}=0.402$. Theoretical first-principle calculations on dysprosium metal are needed for the proposed crystal structures in order to confirm their stability and establish the electronic structure at high compressions.

\section{ACKNOWLEDGEMENTS}

This work was performed under the auspices of the U.S. Department of Energy by the 
University of California, Lawrence Livermore National Laboratory under contract No. W-7405-

Eng-48. Funding for this research was provided by the LLNL B-Program and we thank Dr. Larry Wiley for his encouragement. We benefited from technical discussions we had with Professor Y. K. Vohra of the University of Alabama at Birmingham, and we appreciate his helpful suggestions. We also appreciate the help and technical assistance provided by Dr. Jingzhu Hu while at the NSLS hutch X-17C. 


\section{REFERENCES}

${ }^{1}$ A. Jayaraman, Phys. Rev. 135, A1056 (1964), and A. Jayaraman, in Handbook on the Physics and Chemistry of the Rare Earths, Vol. 1 Ch. 9, edited by K.A. Gschneider and L. Eyring (North-Holland, Amsterdam, 1978).

${ }^{2}$ W. B. Holzapfel, J. Alloys Compd. 223, 170 (1995).

${ }^{3}$ B. Johansson, Philos. Mag. 30, 469 (1974).

${ }^{4}$ J. Donohue, The Structures of the Elements (Robert E. Krieger, Florida, 1982), p. 109.

${ }^{5}$ W. A. Großhans, Röntgenbeugung an einigen Seltenen Erden unter Druck, Ph. D. Thesis (Univ. of Paderborn, Germany, 1987) p. A1-27.

${ }^{6}$ J. Akella, G.S. Smith, S.T. Weir, and C. Ruddle, in High Pressure Science and Technology, edited by W. A. Trzeciakowski, XV AIRAPT Conf. Proc., Warsaw, Poland (World Science, 1995) p. 387.

${ }^{7}$ P. Vinet, J. Ferrante, J.H. Rose, and J.R. Smith, J. Geophys. Res. 92, 9319 (1987).

${ }^{8}$ S.K. Sikka, Phys. Rev. B 38, 8463 (1988).

${ }^{9}$ R.G. McQueen, S.P. Marsh, J.W. Taylor, J.M. Fritz, and W.J. Carter, in High-Velocity Impact Phenomena, edited by R. Kinslow (Academic, New York, 1970) Ch. VII.

${ }^{10}$ N. Hamaya, Y. Sakamoto, H. Fujihisa, Y. Fujii, K. Takemura, T. Kikegawa, and O. Shimomura, in High Pressure Science and Technology, edited by S.C. Schmidt et al., AIP Conf. Proc. No. 309 (AIP, New York, 1994) p. 457.

${ }^{11}$ W.H. Zachariasen and F.H. Ellinger, Acta Cryst. A33, 155 (1977).

${ }^{12}$ H. Hua, Y.K. Vohra, J. Akella, S.T. Weir, R. Ahuja, and B. Johansson, Rev. High Pressure Sci. Technol. 7, 233 (1998). 
FIGURE 1

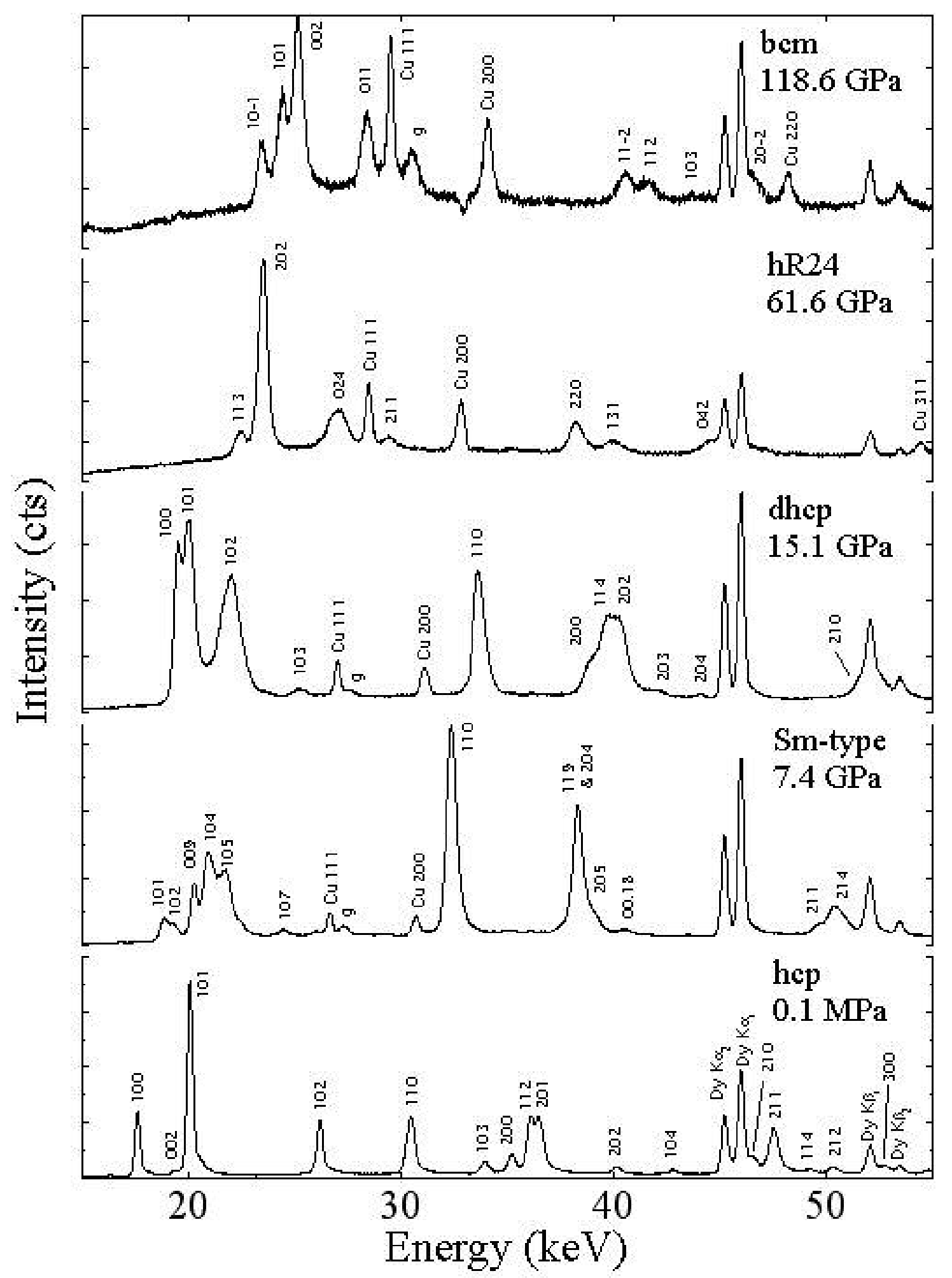




\section{FIGURE 2}

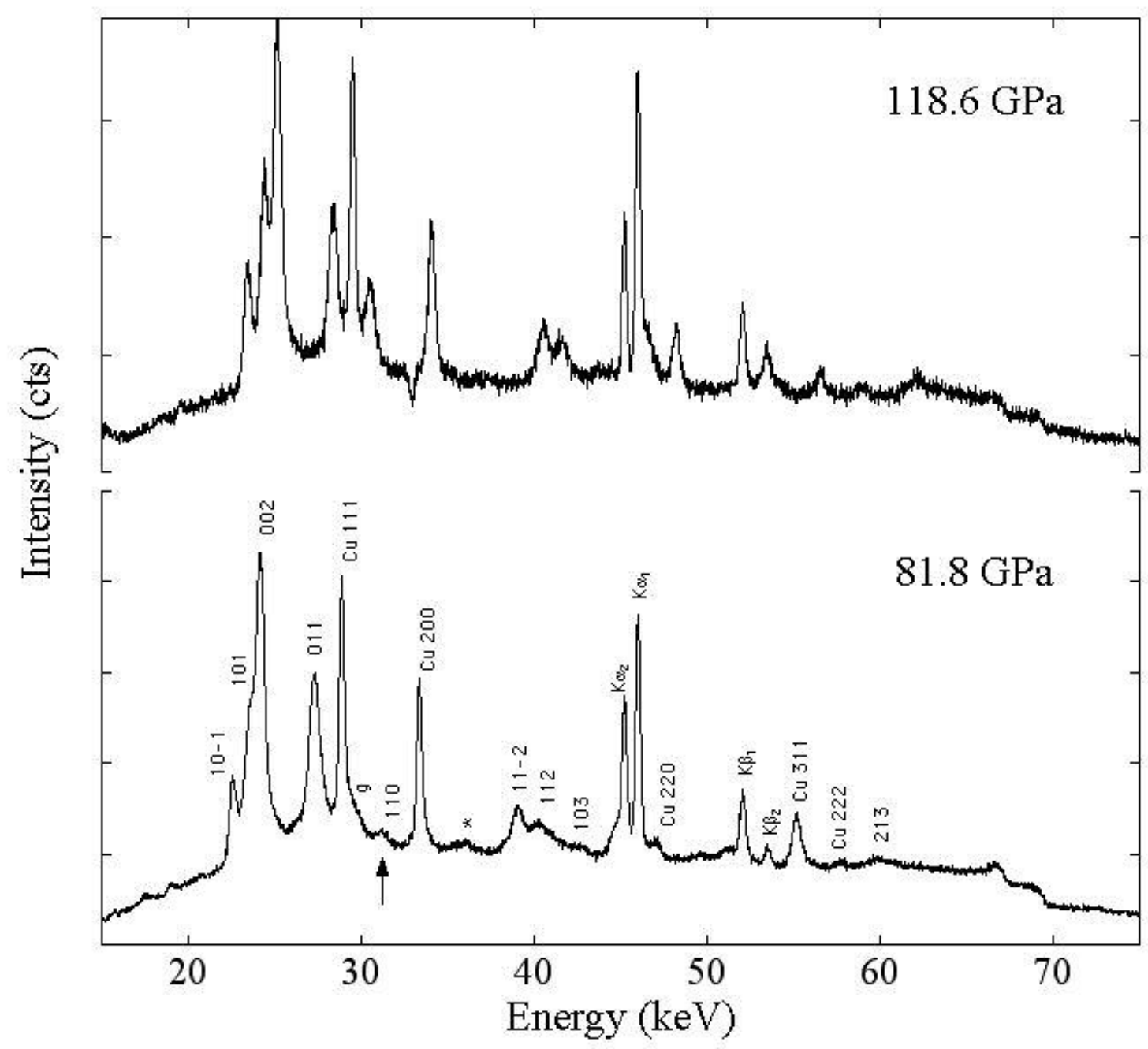


FIGURE 3

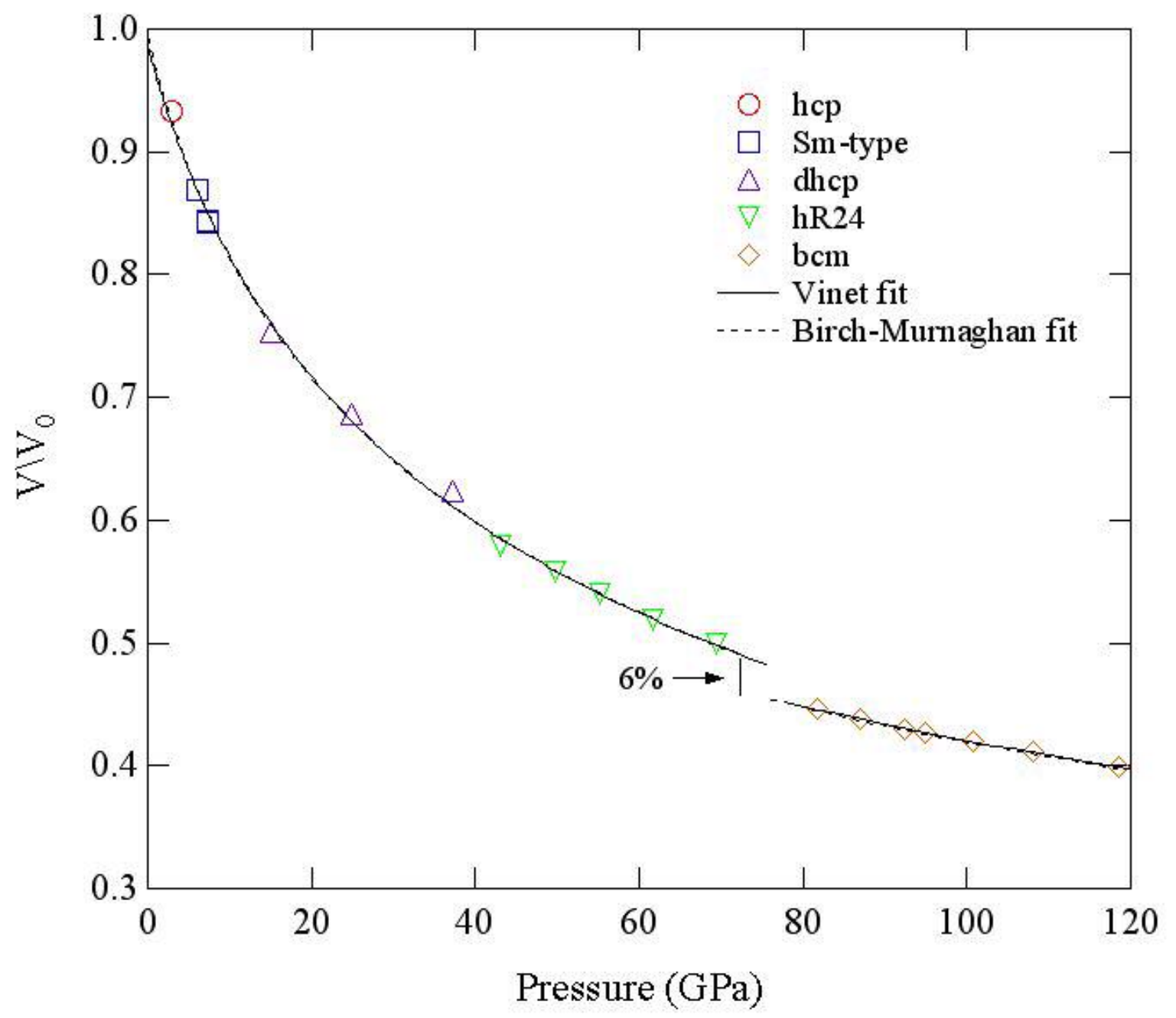


TABLE I. Observed and calculated d-spacings and intensities for the hR24 phase of Dy at 61.6 GPa. The sample shows evidence of preferred orientation as seen in the discrepancy between the observed and calculated intensities.

\begin{tabular}{ccccc}
\hline \hline$h k l$ & $d_{\text {obs }}$ & $d_{\text {calc }}$ & $I_{\text {obs }}$ & $I_{\text {calc }}$ \\
\hline 113 & 2.4452 & 2.4320 & 0.52 & 2.2 \\
202 & 2.3271 & 2.3283 & 4.0 & 100 \\
024 & 2.0230 & 2.0166 & 100 & 85.0 \\
211 & 1.8569 & 1.8504 & 19.4 & 2.3 \\
033 & 1.5588 & 1.5522 & 18.3 & 4.4 \\
220 & 1.4301 & 1.4258 & 70.0 & 39.3 \\
042 & 1.2295 & 1.2159 & 24.1 & 17.1 \\
422 & 0.9306 & 0.9252 & 11.2 & 7.7 \\
244 & 0.8861 & 0.9018 & 11.5 & 6.5 \\
\hline \hline
\end{tabular}

TABLE II. Observed and calculated d-spacings and intensities for body-centered monoclinic Dy at 118.6 GPa. Sample texturing is still prominent as seen in the intensities as well as the absence of the 110 peak.

\begin{tabular}{ccccc}
\hline \hline$h k l$ & $d_{\text {obs }}$ & $d_{\text {calc }}$ & $I_{\text {obs }}$ & $I_{\text {calc }}$ \\
\hline $10-1$ & 2.3351 & 2.3361 & 4.7 & 32.9 \\
101 & 2.2425 & 2.2428 & 7.1 & 37.8 \\
002 & 2.1744 & 2.1745 & 14.3 & 41.3 \\
011 & 1.9272 & 1.9276 & 100 & 100 \\
$11-2$ & 1.3495 & 1.3480 & 36.0 & 64.3 \\
112 & 1.3137 & 1.3114 & 23.1 & 59.2 \\
$20-2$ & 1.1695 & 1.1680 & 36.5 & 19.5 \\
213 & 0.8807 & 0.8800 & 10.1 & 62.1 \\
\hline \hline
\end{tabular}




\section{FIGURE CAPTIONS}

FIG. 1. EDXD spectra of Dy on compression. All observed phases of Dy to $119 \mathrm{GPa}$ are shown in this figure along with the (hkl) assignments of various diffraction peaks in each spectrum.

FIG. 2. Diffraction spectra of body-centered monoclinic Dy in the ultra high-pressure range between $81 \mathrm{GPa}$ and $119 \mathrm{GPa}$. The diamond absorption edge becomes apparent in the spectrum at $119 \mathrm{GPa}$ obscuring the weak (110) diffraction peak from the sample.

FIG. 3. The measured equation of state for various phases of Dy to $119 \mathrm{GPa}$ at room temperature. The solid curves are the fits to the Birch-Murnaghan and Vinet equations described in the text. The volume change of $6 \%$ at the $73 \mathrm{GPa}$ phase transition is clearly evident. 